\title{
Multiple factors affect pest and pathogen damage on 31 Populus clones in South Carolina ${ }^{\text {is }}$
}

\author{
David R. Coyle ${ }^{\mathrm{a}, *}$, Mark D. Coleman ${ }^{\mathrm{a}}$, Jaclin A. Durant ${ }^{\mathrm{b}, 1}$, Lee A. Newman ${ }^{\mathrm{b}, \mathrm{c}}$ \\ ${ }^{a}$ USDA Forest Service, Southern Research Station, P.O. Box 700, New Ellenton, SC 29809, USA \\ ${ }^{\mathrm{b}}$ Arnold School of Public Health, Department of Environmental Health Sciences, University of South Carolina, 800 Sumter St., Columbia, SC 29208, USA \\ ${ }^{\mathrm{c}}$ University of Georgia Savannah River Ecology Laboratory, Drawer E, Aiken, SC 29802, USA
}

Received 10 January 2005; received in revised form 8 July 2005; accepted 22 July 2005

Available online 19 April 2006

\begin{abstract}
Populus species and hybrids have many practical applications, but there is a paucity of data regarding selections that perform well in the southeastern US. We compared pest susceptibility of 31 Populus clones over 3 years in South Carolina, USA. Cuttings were planted in spring 2001 on two study sites. Clones planted in the bottomland site received granular fertilizer yearly and irrigation the first two years only, while those on the sandy, upland site received irrigation and fertilization throughout each growing season. Foliar damage by the cottonwood leaf beetle (Chrysomela scripta), cottonwood leafcurl mite (Tetra lobulifera), and poplar leaf rust (Melampsora medusae) was visually monitored several times each growing season. Damage ratings differed significantly among clones, and clonal rankings changed from year to year. Irrigation increased $C$. scripta and $M$. medusae damage, but had no effect on T. lobulifera damage. Certain clones received greater pest damage at a particular study site. Temporal damage patterns were evident among individual clones and on each site. At the upland site, OP367 and 7300502 were highly resistant to all three pests; I45/51 was highly resistant to $C$. scripta and $M$. medusae; NM6 and 15-29 were highly resistant to M. medusae; and 7302801 was highly resistant to T. lobulifera and M. medusae. At the bottomland site, NM6, Eridano, I45/51, and 7302801 were highly resistant to all three pests; clone 7300502 was highly resistant to $M$. medusae only. Based on this preliminary 3-year study of pest damage levels, we would recommend clones NM6, Eridano, I45/51, OP367, 15-29, 7302801, 7300502, and Kentucky 8 for use in this region.
\end{abstract}

Published by Elsevier Ltd.

Keywords: Chrysomela scripta; Clone; Irrigation; Melampsora medusae; Temporal variation; Tetra lobulifera

\section{Introduction}

With production rates far greater than could be obtained naturally, wood and wood products from intensively managed forest plantations may reduce harvest pressure

This research was conducted on official Government time, and therefore the right of the US Government to retain copyright is accepted. The use of trade names in this publication is solely for reader information and does not imply endorsement by the US Department of Agriculture or the University of South Carolina.

*Corresponding author. Current address: Department of Entomology, 345 Russell Laboratories, University of Wisconsin, Madison, WI 53706, USA. Tel.: + 16082624755 ; fax: + 16082623322 .

E-mail address: dcoyle@entomology.wisc.edu (D.R. Coyle).

${ }^{1}$ Current address: Department of Entomology, University of CaliforniaRiverside, Riverside, CA 92521, USA. on native forests [1,2]. Additionally, species such as Populus [3] and Salix [4] can be used for phytoremediation, carbon sequestration, erosion control, and biomass production.

Genotypes of Populus are desirable for intensive management given their rapid growth characteristics and established propagation methodologies [5]. A major focus of Populus breeding programs is on producing highyielding genotypes [6]. If not careful in selection schemes there is a potential loss of pest resistance or tolerance if the breeding strategy focuses too strictly on growth alone [7,8]. Growth is the most common variable used in the selection process, but mortality [9], rootability [10], and pest susceptibility [11-13] should also be included among the selection criteria.

The monocultural environment created by intensive management creates an ideal situation for pest infestations 
[14,15]. Populus selections are particularly susceptible to a suite of insect and disease pests [16-19]. Three pests are of major importance in the southeastern US: the cottonwood leaf beetle, Chrysomela scripta F. (Coleoptera: Chrysomelidae), the cottonwood leafcurl mite, Tetra lobulifera (Keifer) (Acari: Eriophyidae), and leaf rust, Melampsora medusae Thuem. (Basidiomycetes: Uredinales).

C. scripta is the most economically important pest of intensively managed Populus in the eastern US [19]. This multivoltine folivore can cause extensive leaf loss [20], and C. scripta feeding has been shown to reduce stem volume by over $70 \%$ in plantation-grown Populus [21]. T. lobulifera is cosmopolitan over much of the US [22] and can be a serious pest in the Southeast [23]. Certain Populus clones have been removed from commercial production due to high susceptibility to $T$. lobulifera (RJ Rousseau, pers. commun.). Melampsora leaf rust is a potentially lethal disease to young Populus [24-26]. Its alternate host lifestyle and lack of viable control tactic has made breeding rust resistance into new Populus selections a priority [16,25].

Several Populus selection trials have taken place in the Mississippi Delta region [27,28], but virtually no published information exists regarding Populus pest susceptibility in the southeastern US coastal plain. Our objectives were to evaluate pest resistance among a range of top performing Populus clones in the southeastern US. We visually measured leaf damage on several selections from the southeastern, northwestern, and north-central US on two contrasting study sites in South Carolina. We hypothesized that (1) clones would differ in their susceptibility to pest damage, (2) increased pest damage would occur on trees receiving irrigation, (3) pest damage would differ between study sites, and (4) pest damage would exhibit significant temporal variation over the course of a growing season.

\section{Materials and methods}

\subsection{Study location}

The trial was conducted on two sites (termed D-Area and SRWC) located on the US Department of Energy Savannah River Site, a National Environmental Research Park, near Aiken, SC, USA $\left(33^{\circ} 23^{\prime} \mathrm{N}, 81^{\circ} 40^{\prime}\right.$ E). The climate is humid continental, with warm, dry summers and mild winters; temperature varied little between the sites and years, but mean annual rainfall was slightly greater at DArea. Additional site details can be found in WSRC [29], Coleman et al. [30], and Coyle et al. [31].

\subsection{Plant material}

A total of 18 and 31 pure species and hybrid Populus clones were used at D-Area and SRWC, respectively [31]. Clones chosen represented a range of growing regions and genotypes, with particular emphasis on those reported or expected to do well in the southeastern US. Dormant hardwood cuttings were stored at $3{ }^{\circ} \mathrm{C}$ and soaked in water $48 \mathrm{~h}$ prior to planting to promote optimum rooting [32].

\subsection{Study design and treatments}

Specific details are available in Coyle et al. [31]; a brief description follows. Both sites were planted in spring 2001; D-Area with four blocks of 18 clones at $1.3 \times 1.3 \mathrm{~m}$ spacing, and SRWC with two blocks of 31 clones at $2.5 \times 2.5 \mathrm{~m}$ spacing. D-Area clones $\mathrm{S} 7 \mathrm{C} 15,52-225$, and Eridano were planted in 36-tree plots; all others were planted in 16-tree plots. Two blocks at D-Area received irrigation during the 2001 and 2002 growing seasons at a rate of 1.5 and $2.0 \mathrm{~cm} \mathrm{wk}^{-1}$, respectively, while control blocks received $0.1 \mathrm{~cm} \mathrm{wk}^{-1}$ irrigation. Trees received only rainwater in 2003. Granular fertilizer $\left(112 \mathrm{~kg} \mathrm{~N} \mathrm{ha}^{-1}\right)$ plus micronutrients was applied to D-Area blocks each spring. SRWC clones 110531, 112127, ST70, ST71, ST109, and ST260 were planted in eight-tree plots due to material availability; all others were planted in 16-tree plots. Trees at SRWC received $3.0 \mathrm{~cm} \mathrm{wk}^{-1}$ irrigation and were fertilized at a rate of $160 \mathrm{~kg} \mathrm{Nha}^{-1}$ annually. Both study sites were kept weed free throughout the experiment.

\subsection{Pest damage assessment}

Pest damage was visually monitored several times each growing season on the interior four trees per clonal plot. Where a clonal plot was comprised of only eight individuals (two rows of four trees), the center four trees were monitored. We recorded $C$. scripta defoliation using a 0-4 rating scale [33] where $0=$ no $C$. scripta defoliation and $4=$ severe $(>75 \%)$ defoliation and/or terminal mortality on leaf plastochron index (LPI) $0-8$. The LPI is a leaf-numbering system whereby the most apical leaf on a terminal or branch with a lamina length of $\geqslant 3.0 \mathrm{~cm}$ is termed "LPI 0". Leaves are numbered positively moving toward the stem and negatively away from the stem [34]. $C$. scripta damage was recorded every year on both sites. $T$. lobulifera damage was recorded on LPI 0-12 using a 0-6 rating scale, where $0=$ no $T$. lobulifera damage and $6=$ extreme foliar curl and discoloration, $>75 \%$ defoliation, or a dead terminal [23]. T. lobulifera damage was recorded in 2001 and 2002 at D-Area, and in 2002 at SRWC. Leaf rust was assessed using the Schreiner [35] scale, where values are assigned based on rust intensity and percent infestation; the values are then multiplied to obtain a final damage rating. M. medusae damage was recorded every year at D-Area, and at SRWC in 2002 and 2003.

\subsection{Statistical analyses}

Pest damage ratings were analyzed separately each season using a repeated measures analysis of variance (Proc MIXED; SAS Inc., Cary, NC). Damage ratings were recorded at different time intervals each growing season; 
therefore, no comparisons between years were made. Means were compared using the Tukey's $t$-test $(\alpha=0.05)$.

At D-Area, we were most interested in the main effects of clone, irrigation, and time. The clone $\times$ irrigation, clone $\times$ time, and clone $\times$ irrigation $\times$ time interactions also were examined to determine if irrigated clones received less pest damage and to determine if pest damage varied over the course of a growing season. Since all trees at the SRWC site received the same treatment, we were only interested in the effects of clone, time, and the clone $\times$ time interaction. Finally, we compared 2003 C. scripta and M. medusae damage on clones at D-Area receiving the irrigation treatment with the same clones at SRWC (hereafter referred to as "common clones"; see [31]) to examine the effects of site, clone, and the site $\times$ clone interaction on damage levels.

A single control S7C1 and D105 clone survived at DArea; these clones are not included in any analysis, but data are presented for comparison. By 2003, the only WV416 clones surviving at D-Area were in irrigated plots; therefore, this clone is not included in the $2003 \mathrm{D}$-Area analysis. At SRWC, no S7C1 or 112830 clones survived; thus they are not included in any analysis. Data from D105 was used only in the 2002 analysis, as this clone experienced $100 \%$ mortality by 2003 .

\section{Results}

\subsection{Clone effects}

Damage by $C$. scripta, T. lobulifera, and M. medusae varied significantly among clones each year at both study sites (Table 1). Overall, damage at both sites was consistent over three years, as indicated by the small changes in clonal rankings (Tables 2 and 3). Certain clones did, however, experience dramatic changes in damage rankings. For instance, C. scripta damage rankings at SRWC increased greatly on Eridano and 184-411 from 2002 to 2003 (Table 3).

\subsection{Irrigation effects}

Irrigation had a significant effect on pest damage ratings (Table 1). Greater C. scripta damage occurred on irrigated trees every year (Fig. 1). A significant clone $\times$ irrigation interaction occurred for C. scripta in 2001 and 2003, while a marginally significant interaction occurred in 2002 (Table 1). Greater C. scripta defoliation occurred on irrigated clones 110804 and S7C15 every year, whereas the irrigated NM6 trees had significantly less defoliation each year. Irrigated clone 7300502 had significantly less defoliation than control trees in 2002 and 2003. Kentucky 8 had greater $C$. scripta damage on trees receiving irrigation in 2003 .

Irrigation alone had no effect on $T$. lobulifera damage, but the clone $\times$ irrigation interaction was significant each year (Table 1). No effect was observed in most clones; however, significantly less $T$. lobulifera damage occurred on clones 110804 and ST66 receiving irrigation in 2001 and 2002 (data not shown).

M. medusae damage was greatest on irrigated trees each year (Fig. 1), and damage was significantly affected by the clone $\times$ irrigation interaction (Table 1). Nearly all clones receiving irrigation showed increased $M$. medusae foliar

Table 1

ANOVA statistics for pest damage ratings during a 3-yr Populus clone trial in South Carolina

\begin{tabular}{|c|c|c|c|c|c|c|c|}
\hline \multirow[t]{2}{*}{ Pest } & \multirow[t]{2}{*}{ Source } & \multicolumn{3}{|c|}{ D-Area } & \multicolumn{3}{|c|}{ SRWC } \\
\hline & & 2001 & 2002 & 2003 & 2001 & 2002 & 2003 \\
\hline \multirow[t]{6}{*}{ C. scripta } & Clone & $* * * *$ & $* *$ & $* * * *$ & $\mathrm{dnr}$ & $* * * *$ & $* * * *$ \\
\hline & Irrigation & $* * * *$ & $* * *$ & $* * * *$ & $\mathrm{dnr}$ & na & na \\
\hline & Clone $\times$ irrigation & $* * * *$ & $*$ & $* * * *$ & $\mathrm{dnr}$ & na & na \\
\hline & Time & $* * * *$ & $* * * *$ & $* * * *$ & $\mathrm{dnr}$ & $* * * *$ & $* * * *$ \\
\hline & Clone $\times$ time & $* * *$ & $*$ & $* * * *$ & $\mathrm{dnr}$ & $* * * *$ & $* * * *$ \\
\hline & Clone $\times$ irrigation $\times$ time & $\mathrm{ns}$ & ns & $\mathrm{ns}$ & $\mathrm{dnr}$ & na & na \\
\hline \multirow[t]{6}{*}{ T. lobulifera } & Clone & $* * * *$ & $* * * *$ & $\mathrm{dnr}$ & $\mathrm{dnr}$ & $* * * *$ & $\operatorname{dnr}$ \\
\hline & Irrigation & ns & ns & dnr & $\operatorname{dnr}$ & na & dnr \\
\hline & Clone $\times$ irrigation & $* * *$ & $* *$ & $\mathrm{dnr}$ & $\mathrm{dnr}$ & na & $\mathrm{dnr}$ \\
\hline & Time & $* * * *$ & $* * * *$ & dnr & $\mathrm{dnr}$ & $* * * *$ & dnr \\
\hline & Clone $\times$ time & $* * * *$ & $* * * *$ & $\mathrm{dnr}$ & $\mathrm{dnr}$ & $* * * *$ & $\mathrm{dnr}$ \\
\hline & Clone $\times$ irrigation $\times$ time & $* *$ & $* *$ & dnr & $\operatorname{dnr}$ & na & $\operatorname{dnr}$ \\
\hline \multirow[t]{6}{*}{ M. medusae } & Clone & $* * * *$ & $* * * *$ & $* * * *$ & $\mathrm{dnr}$ & $* * * *$ & $* * * *$ \\
\hline & Irrigation & $* * * *$ & $* * *$ & $* * * *$ & $\mathrm{dnr}$ & na & na \\
\hline & Clone $\times$ irrigation & $* * * *$ & $* * *$ & $* * * *$ & $\mathrm{dnr}$ & na & na \\
\hline & Time & $* * * *$ & $* * * *$ & $* * * *$ & $\mathrm{dnr}$ & $* * * *$ & $* * * *$ \\
\hline & Clone $\times$ time & $* * * *$ & $* * * *$ & $* * * *$ & $\operatorname{dnr}$ & $* * * *$ & $* * * *$ \\
\hline & Clone $\times$ irrigation $\times$ time & $* * * *$ & $* * * *$ & $* * * *$ & $\mathrm{dnr}$ & na & na \\
\hline
\end{tabular}

ns, not significant; $* P=0.10>0.05 ; * P=0.05>0.01 ; * * * P=0.01>0.001 ; * * * P<0.001$; na, not applicable; dnr, data not recorded. 
Table 2

Mean pest damage ${ }^{\mathrm{a}}$ and rankings at D-Area over a 3-yr Populus clone trial in South Carolina

\begin{tabular}{|c|c|c|c|c|c|c|c|}
\hline Pest & Clone & 2001 & Rank & 2002 & Rank & 2003 & Rank \\
\hline \multirow[t]{18}{*}{ C. scripta } & S7C1 & 0.20 & 1 & 0.17 & 1 & 0.21 & 1 \\
\hline & 7302801 & 0.24 & 2 & 0.22 & 2 & 0.22 & 2 \\
\hline & NM6 & 0.37 & 3 & 0.32 & 4 & 0.28 & 3 \\
\hline & WV415 & 0.44 & 4 & 0.28 & 3 & 0.33 & 4 \\
\hline & Eridano & 0.55 & 6 & 0.56 & 11 & 0.39 & 5 \\
\hline & D105 & 0.60 & 7 & 0.45 & 8 & 0.45 & 6 \\
\hline & Kentucky 8 & 0.52 & 5 & 0.33 & 5 & 0.48 & 7 \\
\hline & $\mathrm{I} 45 / 51$ & 0.68 & 9 & 0.53 & 9 & 0.48 & 8 \\
\hline & WV94 & 0.72 & 10 & 0.38 & 7 & 0.51 & 9 \\
\hline & WV99 & 0.78 & 11 & 0.59 & 14 & 0.51 & 10 \\
\hline & 7300502 & 0.67 & 8 & 0.33 & 6 & 0.53 & 11 \\
\hline & $\mathrm{S} 7 \mathrm{C} 15$ & 0.86 & 14 & 0.58 & 12 & 0.53 & 12 \\
\hline & 110804 & 0.83 & 13 & 0.54 & 10 & 0.58 & 13 \\
\hline & $15-29$ & 0.91 & 16 & 0.64 & 16 & 0.59 & 14 \\
\hline & $\mathrm{S} 13 \mathrm{C} 20$ & 0.80 & 12 & 0.58 & 13 & 0.60 & 15 \\
\hline & WV416 & 0.89 & 15 & 0.64 & 17 & 0.68 & 16 \\
\hline & ST66 & 0.95 & 17 & 0.64 & 15 & 0.68 & 17 \\
\hline & WV316 & 1.43 & 18 & 1.00 & 18 & 0.88 & 18 \\
\hline \multirow[t]{18}{*}{ T. lobulifera } & NM6 & 0.00 & 1 & 0.00 & 1 & & \\
\hline & $\mathrm{I} 45 / 51$ & 0.03 & 2 & 0.03 & 5 & & \\
\hline & Eridano & 0.10 & 6 & 0.13 & 8 & & \\
\hline & $15-29$ & 0.07 & 3 & 0.08 & 7 & & \\
\hline & 7302801 & 0.20 & 9 & 0.25 & 9 & & \\
\hline & D105 & 0.08 & 4 & 0.00 & 2 & & \\
\hline & Kentucky 8 & 0.10 & 7 & 0.03 & 3 & & \\
\hline & $\mathrm{S} 13 \mathrm{C} 20$ & 0.13 & 8 & 0.04 & 6 & & \\
\hline & WV415 & 0.09 & 5 & 0.03 & 4 & & \\
\hline & 7300502 & 0.37 & 15 & 0.25 & 10 & & \\
\hline & $\mathrm{S} 7 \mathrm{C} 1$ & 0.40 & 16 & 0.25 & 13 & & \\
\hline & WV94 & 0.34 & 14 & 0.35 & 16 & & \\
\hline & WV99 & 0.33 & 13 & 0.28 & 15 & & \\
\hline & 110804 & 0.51 & 17 & 0.46 & 18 & & \\
\hline & WV416 & 0.31 & 11 & 0.25 & 11 & & \\
\hline & $\mathrm{S} 7 \mathrm{C} 15$ & 0.32 & 12 & 0.28 & 14 & & \\
\hline & WV316 & 0.23 & 10 & 0.25 & 12 & & \\
\hline & ST66 & 0.67 & 18 & 0.42 & 17 & & \\
\hline \multirow[t]{18}{*}{ M. medusae } & NM6 & 0.01 & 1 & 0.00 & 1 & 0.01 & 1 \\
\hline & $15-29$ & 0.33 & 3 & 0.11 & 3 & 0.19 & 2 \\
\hline & $\mathrm{I} 45 / 51$ & 0.44 & 4 & 0.03 & 2 & 0.27 & 3 \\
\hline & 7302801 & 0.18 & 2 & 0.11 & 4 & 0.47 & 4 \\
\hline & WV416 & 0.84 & 5 & 0.36 & 7 & 0.55 & 5 \\
\hline & Eridano & 1.53 & 6 & 0.17 & 5 & 0.93 & 6 \\
\hline & 7300502 & 4.37 & 7 & 0.33 & 6 & 2.91 & 7 \\
\hline & WV316 & 4.57 & 8 & 0.96 & 9 & 3.05 & 8 \\
\hline & Kentucky 8 & 7.72 & 10 & 1.83 & 12 & 5.65 & 9 \\
\hline & ST66 & 9.67 & 12 & 1.75 & 11 & 6.49 & 10 \\
\hline & $\mathrm{S} 7 \mathrm{C} 15$ & 10.04 & 13 & 1.18 & 10 & 6.71 & 11 \\
\hline & 110804 & 9.66 & 11 & 4.93 & 14 & 6.85 & 12 \\
\hline & $\mathrm{S} 7 \mathrm{C} 1$ & 13.07 & 15 & 5.92 & 15 & 8.17 & 13 \\
\hline & $\mathrm{S} 13 \mathrm{C} 20$ & 12.47 & 14 & 0.88 & 8 & 8.31 & 14 \\
\hline & D105 & 5.36 & 9 & 4.80 & 13 & 10.48 & 15 \\
\hline & WV99 & 16.93 & 17 & 12.75 & 18 & 11.59 & 16 \\
\hline & WV415 & 17.40 & 18 & 8.47 & 17 & 13.59 & 17 \\
\hline & WV94 & 14.58 & 16 & 5.98 & 16 & 14.44 & 18 \\
\hline
\end{tabular}

${ }^{\text {a }}$ Damage rating scale for $C$. scripta was from 0 to 4 on LPI 0 to 8 , for $T$. lobulifera was from 0 to 6 on LPI 0 to 12, and for M. medusae was from 0 to 100 based on percent foliage infested and infestation severity.
Table 3

Mean pest damage a and rankings at SRWC over a 3-yr Populus clone trial in South Carolina

\begin{tabular}{|c|c|c|c|c|c|}
\hline Pest & Clone & 2002 & Rank & 2003 & Rank \\
\hline \multirow[t]{31}{*}{ C. scripta } & OP367 & 0.00 & 1 & 0.95 & 1 \\
\hline & $\mathrm{I} 45 / 51$ & 0.00 & 1 & 1.06 & 2 \\
\hline & $311-93$ & 0.00 & 1 & 1.13 & 3 \\
\hline & 7300502 & 0.36 & 5 & 1.20 & 4 \\
\hline & ST71 & 0.58 & 11 & 1.22 & 5 \\
\hline & ST261 & 0.43 & 8 & 1.23 & 6 \\
\hline & WV94 & 0.68 & 13 & 1.28 & 7 \\
\hline & $\mathrm{S} 13 \mathrm{C} 20$ & 0.50 & 9 & 1.33 & 8 \\
\hline & ST109 & 0.76 & 14 & 1.35 & 9 \\
\hline & $15-29$ & 0.32 & 4 & 1.38 & 10 \\
\hline & Kentucky 8 & 0.90 & 16 & 1.38 & 10 \\
\hline & $\mathrm{S} 7 \mathrm{C} 15$ & 0.37 & 6 & 1.43 & 11 \\
\hline & $52-225$ & 1.00 & 17 & 1.46 & 12 \\
\hline & ST260 & 0.50 & 9 & 1.50 & 13 \\
\hline & WV415 & 0.53 & 10 & 1.50 & 13 \\
\hline & ST66 & 0.60 & 12 & 1.50 & 13 \\
\hline & 112127 & 1.20 & 20 & 1.50 & 13 \\
\hline & NM6 & 0.06 & 2 & 1.54 & 14 \\
\hline & $184-411$ & 0.27 & 3 & 1.60 & 15 \\
\hline & WV416 & 0.50 & 9 & 1.61 & 16 \\
\hline & 110531 & 0.77 & 15 & 1.65 & 17 \\
\hline & WV316 & 1.18 & 19 & 1.65 & 17 \\
\hline & 110804 & 0.60 & 12 & 1.67 & 18 \\
\hline & Eridano & 0.40 & 7 & 1.70 & 19 \\
\hline & WV99 & 1.23 & 21 & 1.79 & 20 \\
\hline & 7302801 & 1.05 & 18 & 1.84 & 21 \\
\hline & ST70 & 1.40 & 22 & 2.00 & 22 \\
\hline & ST264 & 1.80 & 23 & 2.03 & 23 \\
\hline & $\mathrm{D} 105^{\mathrm{b}}$ & 0.40 & 7 & na & \\
\hline & $\mathrm{S} 7 \mathrm{Cl}^{\mathrm{c}}$ & na & na & na & \\
\hline & $112830^{\mathrm{c}}$ & na & na & na & \\
\hline \multirow[t]{31}{*}{ T. lobulifera } & 7302801 & 0.25 & 1 & & \\
\hline & OP367 & 0.25 & 1 & & \\
\hline & ST109 & 0.28 & 2 & & \\
\hline & WV99 & 0.30 & 3 & & \\
\hline & $52-225$ & 0.30 & 3 & & \\
\hline & $\mathrm{S} 7 \mathrm{C} 15$ & 0.31 & 4 & & \\
\hline & ST264 & 0.32 & 5 & & \\
\hline & $184-411$ & 0.33 & 6 & & \\
\hline & 7300502 & 0.36 & 7 & & \\
\hline & D105 & 0.40 & 8 & & \\
\hline & $15-29$ & 0.40 & 8 & & \\
\hline & 112127 & 0.40 & 8 & & \\
\hline & ST260 & 0.40 & 8 & & \\
\hline & ST261 & 0.43 & 9 & & \\
\hline & WV94 & 0.44 & 10 & & \\
\hline & WV415 & 0.47 & 11 & & \\
\hline & $\mathrm{I} 45 / 51$ & 0.48 & 12 & & \\
\hline & ST66 & 0.50 & 13 & & \\
\hline & Eridano & 0.50 & 13 & & \\
\hline & ST71 & 0.50 & 13 & & \\
\hline & 110804 & 0.53 & 14 & & \\
\hline & WV316 & 0.55 & 15 & & \\
\hline & 110531 & 0.55 & 15 & & \\
\hline & $\mathrm{S} 13 \mathrm{C} 20$ & 0.56 & 16 & & \\
\hline & ST70 & 0.60 & 17 & & \\
\hline & NM6 & 0.66 & 18 & & \\
\hline & Kentucky 8 & 0.80 & 19 & & \\
\hline & WV416 & 0.83 & 20 & & \\
\hline & $311-93$ & 1.10 & 21 & & \\
\hline & $\mathrm{S} 7 \mathrm{C} 1^{\mathrm{c}}$ & na & & & \\
\hline & $112830^{\mathrm{c}}$ & na & & & \\
\hline
\end{tabular}


Table 3 (continued)

\begin{tabular}{|c|c|c|c|c|c|}
\hline Pest & Clone & 2002 & Rank & 2003 & Rank \\
\hline \multirow[t]{31}{*}{ M. medusae } & NM6 & 0.0 & 1 & 0.0 & 1 \\
\hline & $15-29$ & 0.0 & 1 & 0.0 & 1 \\
\hline & $184-411$ & 0.0 & 1 & 0.0 & 1 \\
\hline & $311-93$ & 0.0 & 1 & 0.0 & 1 \\
\hline & $52-225$ & 0.0 & 1 & 0.0 & 1 \\
\hline & $\mathrm{I} 45 / 51$ & 0.0 & 1 & 0.1 & 2 \\
\hline & 7302801 & 0.0 & 1 & 0.2 & 3 \\
\hline & OP367 & 0.1 & 2 & 0.3 & 4 \\
\hline & ST70 & 0.0 & 1 & 0.6 & 5 \\
\hline & WV416 & 0.0 & 1 & 0.9 & 6 \\
\hline & 7300502 & 3.4 & 4 & 2.8 & 7 \\
\hline & Eridano & 4.5 & 5 & 3.0 & 8 \\
\hline & ST261 & 4.7 & 6 & 3.6 & 9 \\
\hline & S7C15 & 2.8 & 3 & 3.7 & 10 \\
\hline & ST66 & 7.5 & 8 & 6.3 & 11 \\
\hline & $\mathrm{S} 13 \mathrm{C} 20$ & 7.2 & 7 & 6.7 & 12 \\
\hline & 110531 & 10.2 & 10 & 7.5 & 13 \\
\hline & ST71 & 9.3 & 9 & 8.1 & 14 \\
\hline & WV99 & 16.8 & 12 & 11.7 & 15 \\
\hline & ST264 & 17.4 & 13 & 11.9 & 16 \\
\hline & Kentucky 8 & 15.8 & 11 & 12.0 & 17 \\
\hline & ST260 & 30.3 & 14 & 20.3 & 18 \\
\hline & WV316 & 32.7 & 15 & 22.6 & 19 \\
\hline & 112127 & 33.0 & 16 & 23.4 & 20 \\
\hline & WV94 & 35.9 & 17 & 26.0 & 21 \\
\hline & WV415 & 36.7 & 18 & 28.3 & 22 \\
\hline & 110804 & 49.0 & 19 & 32.3 & 23 \\
\hline & ST109 & 52.2 & 20 & 36.1 & 24 \\
\hline & $\mathrm{D} 105^{\mathrm{b}}$ & 55.0 & 21 & na & \\
\hline & $\mathrm{S} 7 \mathrm{Cl}^{\mathrm{c}}$ & na & & na & \\
\hline & $112830^{c}$ & na & & na & \\
\hline
\end{tabular}

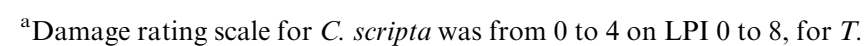
lobulifera was from 0 to 6 on LPI 0 to 12, and for M. medusae was from 0 to 100 based on percent foliage infested and infestation severity.

${ }^{\mathrm{b}}$ Clone D105 suffered $100 \%$ mortality prior to the 2003 growing season.

${ }^{c}$ Clones S7C1 and 112830 had $100 \%$ mortality prior to the initiation of pest damage monitoring.

damage. Clones WV94, WV415, and 110814 receiving irrigation all had significantly higher $M$. medusae damage levels each year, whereas clones S7C15, ST66, and Kentucky 8 receiving irrigation had higher damage levels in 2001 and 2003. Clone WV99 had lower M. medusae damage on trees receiving irrigation in 2002.

\subsection{Site effects}

In 2003, mean $C$. scripta damage ratings were significantly greater $(P<0.001)$ at SRWC $(3.13 \pm 0.03)$ compared to D-Area $(2.88 \pm 0.03)$, while $M$. medusae damage did not differ between the sites $(P=0.64)$. Greater $C$. scripta damage ratings on six clones at SRWC compared to DArea were indicated by a significant site $\times$ clone interaction $(P<0.001)$. Three of these clones were hybrids (Eridano, NM6, and 15-29) while three were pure $P$. deltoides (S7C15, WV99, and $\mathrm{S} 13 \mathrm{C} 20$ ). The site $\times$ clone interaction also was significant for $M$. medusae damage $(P<0.001)$;

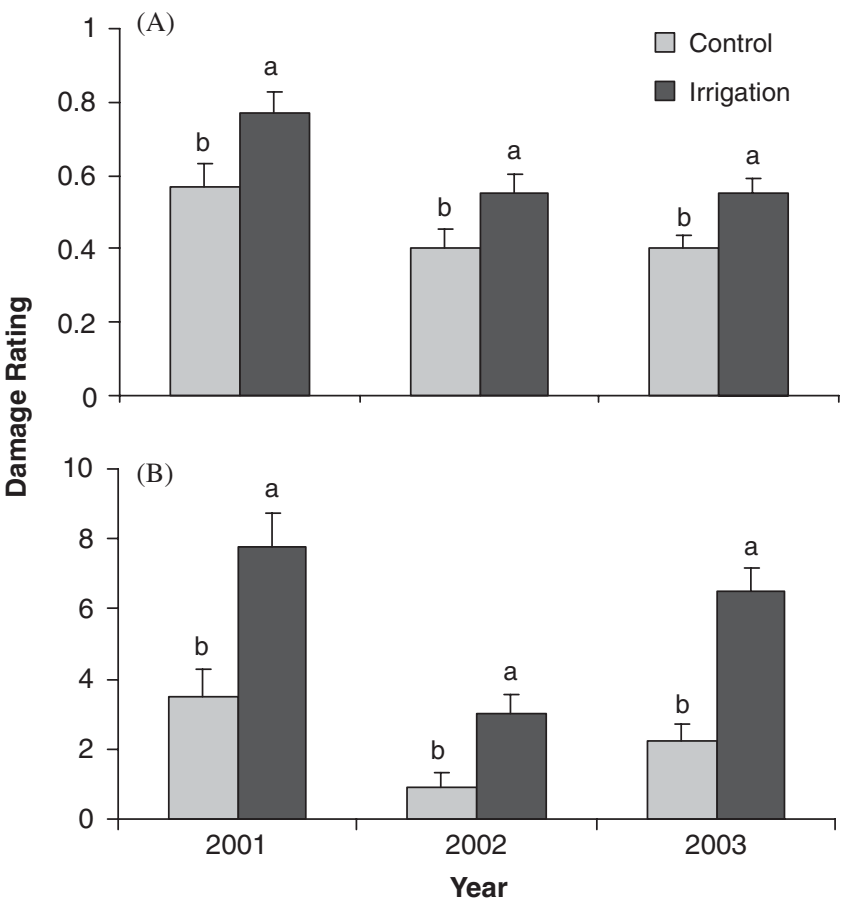

Fig. 1. Effects of irrigation on (A) C. scripta and (B) M. medusae damage levels at D-Area during the 2001-2003 growing seasons. Means $( \pm$ SE) within a combination of pest and year sharing a letter are not significantly different (Tukey's HSD, $\alpha=0.05$ ).

clones WV94 and 7300502 had higher damage ratings at DArea and SRWC, respectively.

\subsection{Time effects}

Within each growing season, pest damage changed significantly at both sites (Table 1). At D-Area, C. scripta and $M$. medusae damage was significantly greater at the end of the 2001 and 2002 growing seasons, but in $2003 C$. scripta and $M$. medusae damage was greatest in mid- and early summer, respectively (Fig. 2). T. lobulifera damage peaked in both mid-summer and late fall during 2001, but only in mid-summer 2002.

At SRWC, C. scripta damage was greatest in August each year, and stayed high through September in 2003 (Fig. 3). In 2002, T. lobulifera damage declined throughout the season until peaking in October (Fig. 3). M. medusae damage increased throughout the 2002 growing season, but peaked in mid-summer 2003 (Fig. 3).

Significant clone $\times$ time interactions occurred at D-Area in 2001 and 2003 for C. scripta, and every year for $T$. lobulifera and $M$. medusae (Table 1). All clone $\times$ time interactions at SRWC were highly significant (Table 1), indicating that pest damage on many clones changed during the growing season. Pest damage on individual clones fell into one of four general patterns throughout a growing season: (1) damage was relatively even (e.g., clones WV316 and 110804), (2) damage began low, then steadily escalated (e.g., clone Kentucky 8), (3) damage peaked once (e.g., clone 184-411), and (4) damage peaked twice (e.g., 


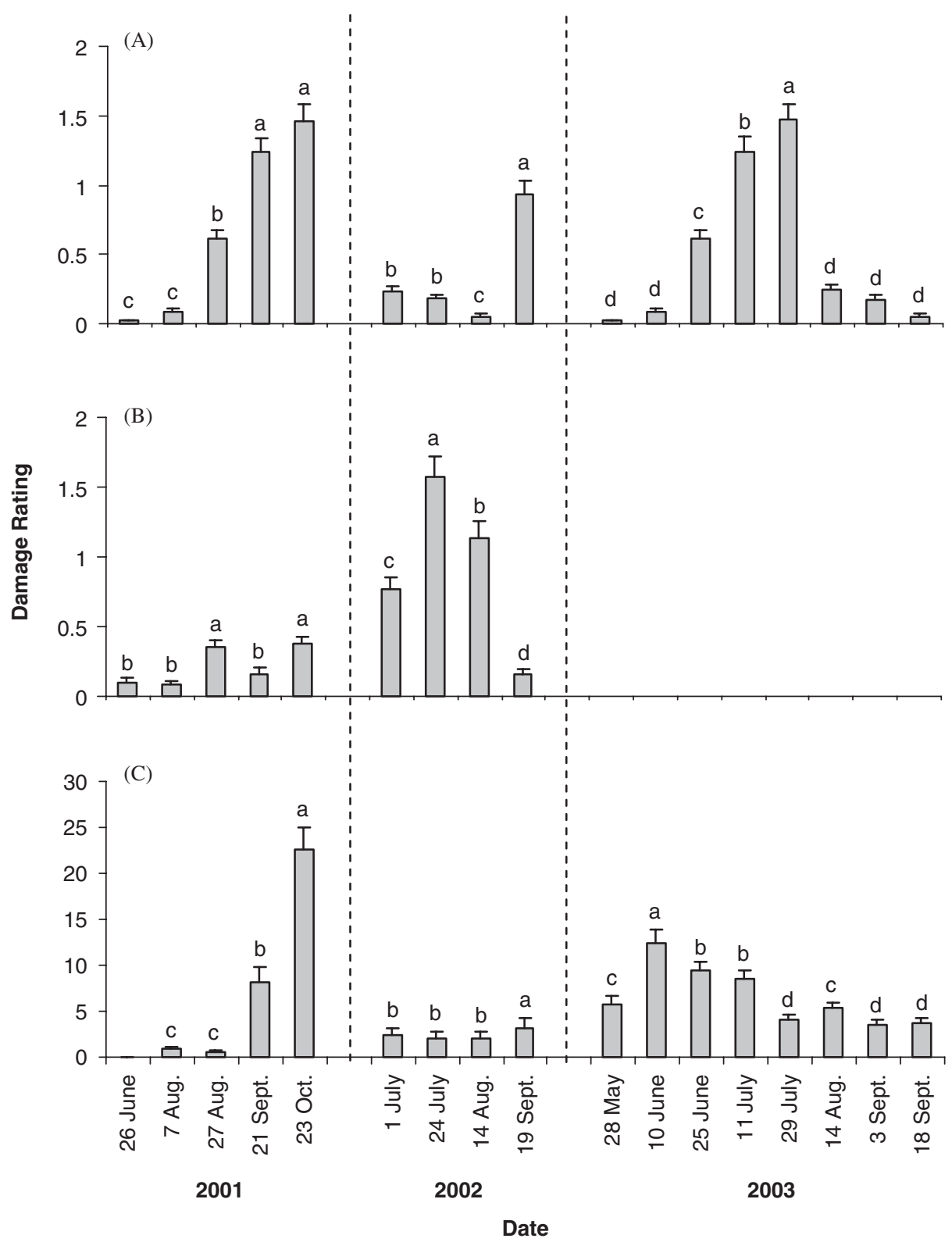

Fig. 2. Temporal pest damage at D-Area from 2001 to 2003: (A) C. scripta, (B) T. lobulifera, (C) M. medusae. Means ( \pm SE) within a year sharing a letter are not significantly different (Tukey's HSD, $\alpha=0.05$ ). Damage ratings for Tetra lobulifera were not recorded in 2003.

clones OP367 and ST70). Clone $\times$ irrigation $\times$ time interactions at D-Area were significant each year for $T$. lobulifera and M. medusae only (Table 1).

\section{Discussion}

\subsection{Clone effects}

Intensively managed Populus plantations are subject to defoliation by a wide variety of pests [19], and a wide range of susceptibility occurs among clones and hybrids [11,12,18,25,36-38]. C. scripta preference [39] and damage [21] varies greatly among Populus clones and hybrids, as does that of T. lobulifera [23] and M. medusae [36,37]. Our study found that several clones of various Populus hybrids and pure $P$. deltoides had low susceptibility to all three pests monitored. In addition, many clones performed very well with respect to two out of three pests (e.g., D105 at DArea and 7302801 at SRWC).

Low overall pest pressure occurred throughout the duration of this study for each of the three pests. Most average $C$. scripta and $T$. lobulifera damage ratings were $<1$ at D-Area and $<2$ at SRWC. Only at SRWC did we see mean rust ratings over 30 . Mean damage ratings $>3$ are common under heavy C. scripta defoliation pressure [20], while a $T$. lobulifera outbreak caused mean damage ratings of almost 4 [23]. Rust damage ratings of 100 have been observed in other studies [40], occasionally resulting in tree mortality [24]. When pest damage levels are low, it can be difficult to determine if certain clones perform better than 


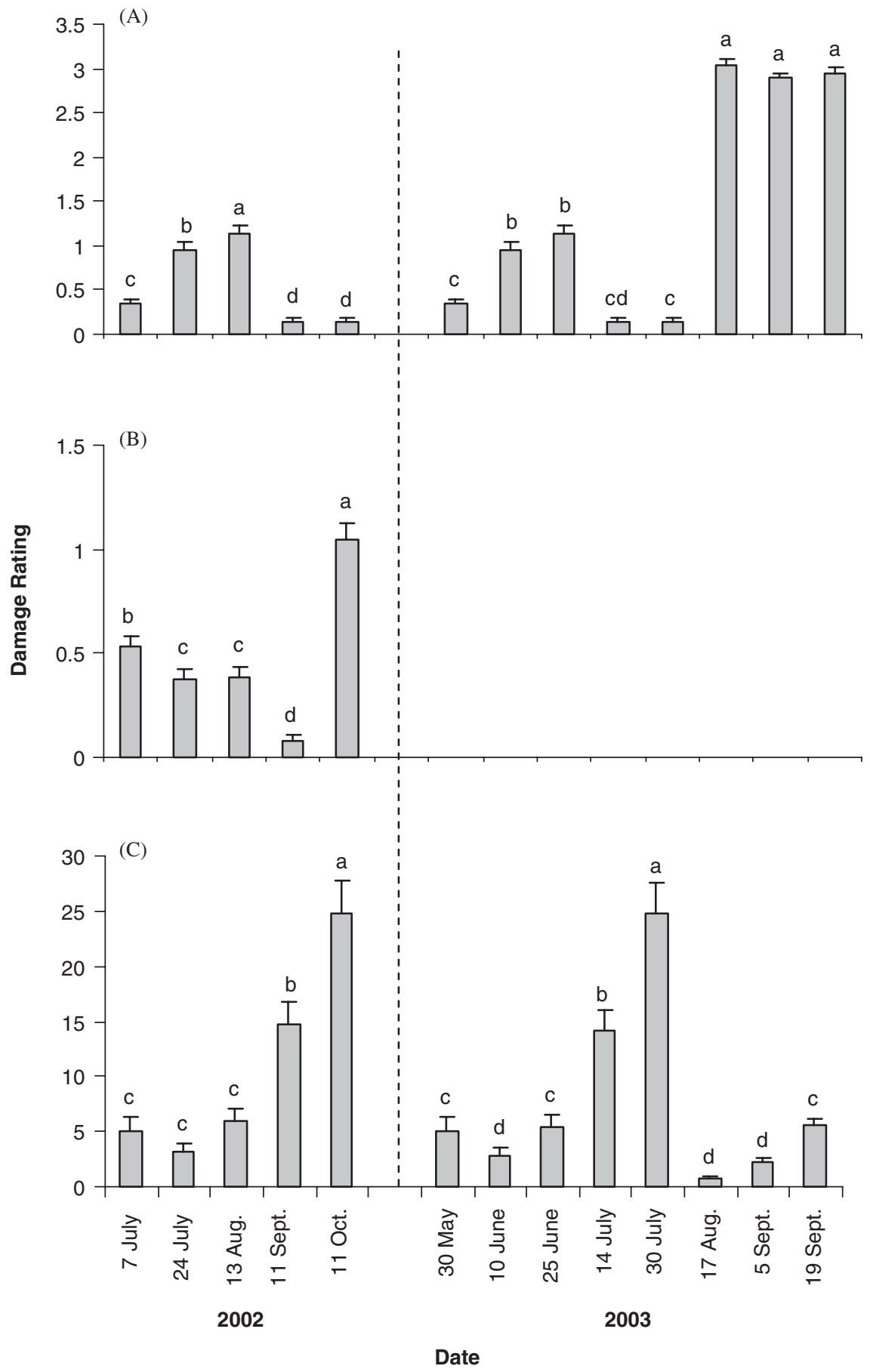

Fig. 3. Temporal pest damage at SRWC from 2002 to 2003: (A) C. scripta, (B) T. lobulifera, (C) M. medusae. T. lobulifera was not monitored in 2003. Means $( \pm$ SE) within a year sharing a letter are not significantly different (Tukey's HSD, $\alpha=0.05$ ). Damage ratings for Tetra lobulifera were not recorded in 2003 .

others; thus greater interpretation is required in these situations.

\subsection{Irrigation effects}

The effect of plant water stress on herbivore performance is an ongoing debate [41-43]. Generally speaking, damage and performance (i.e., body size, fecundity, developmental rate) of folivores is not consistently affected, and that of sap-feeders is reduced on water stressed woody plants. Conversely, fungal pathogens tend to exhibit increased damage and greater performance in moist environments. Results from this study concur with these general trends. Although we did not explicitly measure pest performance, folivore (C. scripta) damage levels were highly variable on irrigated and control trees. Damage by C. scripta was inconsistent within years and among clones. It can be difficult to assess treatment (or in 
our case, clonal) differences when pest populations (and subsequent damage levels) are low as seen previously with the Nantucket pine tip moth, Rhyacionia frustrana (Comstock) (Lepidoptera: Tortricidae) [14]. Daane and Williams [44] found Erythroneura variabilis Beamer (Homoptera: Cicadellidae) leafhopper populations on grapevines linearly related to irrigation amounts. However, we found increased sap-feeder ( $T$. lobulifera) damage on nonirrigated trees in all but two clones. Previous research on Populus near our study site found the effects of irrigation on T. lobulifera damage to be inconsistent [23]. Results from this study do not support the argument that irrigation increases sap-feeder damage. With the exception of clone WV99 in 2002, where significant clone $\times$ irrigation interactions occurred, the highest $M$. medusae damage always occurred on the irrigated trees.

\subsection{Site effects}

Several clones experienced greater $C$. scripta damage at SRWC compared to D-Area. The D-Area site was located in a bottomland area on sandy loam soil, whereas the SRWC site was on an upland sandy site surrounded by a pine forest [30]. Greater beetle recruitment most likely occurred at SRWC due to greater Populus foliage in surrounding plantations, and this may have influenced C. scripta population pressure in our study. Ostry and McNabb [36] found site to be a significant influence on disease occurrence and severity in the north-central US. However, the site did not affect $M$. medusae damage levels, but certain clones were more susceptible at a particular site. Caution should be exercised when interpreting these data, as site comparisons were made during 1 year only.

\subsection{Time effects}

Inter-year variation was small, as clones with low susceptibility remained so throughout the study (especially at D-Area). But some exceptions did occur (e.g., NM6 and Eridano susceptibility to C. scripta at SRWC). These patterns also were seen with canker ratings in Argentina, where some clones remained highly ranked between years 3 and 10 and some changed rankings substantially [45].

Often, pest damage ratings are taken at a single time during a study $[11,46]$ or growing season $[13,37]$. Herbivore damage is known to exhibit temporal variation [47]. Previous work with C. scripta [48], T. lobulifera [23] and M. medusae [24] has indicated changing damage levels over the course of a single growing season. Thus, adequate sampling on a temporal scale must occur to ensure accurate data. Results from our study confirm the importance of taking multiple damage ratings over the course of a season. Single sampling dates may misrepresent what is actually occurring, and consequently may misrepresent clonal performance.

\section{Conclusions}

Based on these preliminary data, hybrid clones NM6, Eridano, I45/51, OP367, and 15-29 and P. deltoides clones 7302801, 7300502, and Kentucky 8 all had generally low pest susceptibility. At SRWC, OP367 and 7300502 were highly resistant to all three pests; I45/51 was highly resistant to C. scripta and M. medusae; NM6 and 15-29 were highly resistant to $M$. medusae; and 7302801 was highly resistant to $T$. lobulifera and $M$. medusae. At DArea, NM6, Eridano, I45/51, and 7302801 were highly resistant to all three pests; clone 7300502 was highly resistant to $M$. medusae only. The effects of irrigation on insect performance and damage were very situational, and results from this study neither disputed nor supported the plant stress hypothesis [43]. However, increased damage ratings by $C$. scripta and $M$. medusae occurred on irrigated clones. Care must be taken to match clones to sites on which their performance will be optimized. Finally, knowledge of temporal variation in pest damage patterns is crucial to accurate pest monitoring.

Ultimately, several variables must be taken into consideration when evaluating and selecting Populus clones for further testing or deployment. We monitored pest susceptibility in this study, and survival and growth previously [31] on these clones. Clones I45/51, Eridano, and NM6 exhibited excellent survival rates [31], and exhibited low pest susceptibility. WV416 grew well at both sites, but was susceptible to $C$. scripta and T. lobulifera. S13C20 and Kentucky 8 grew well at D-Area, and were relatively resistant to all three pests. Hybrids 184-411 and 52-225 grew well at SRWC, and were resistant to T. lobulifera and M. medusae.

M. medusae currently cannot be managed using cultural or chemical techniques; therefore, resistance to this pest is very important. If cultural management strategies were applied for $C$. scripta and T. lobulifera when necessary, clones WV416, S13C20, 184-411, 52-225, and Kentucky 8 may warrant additional evaluation in the southeastern US coastal plain.

\section{Acknowledgements}

We thank the many undergraduate technicians that have assisted with this project: Virginia Slater, Luke Williams, LaDawndrea Priest, Rebecca Rose, Cory Hall, and Ginger Jones (USDA Forest Service-Savannah River), and Jessie Brown, Danny Heenan and Meghan Langley (University of Georgia Savannah River Ecology Laboratory and University of South Carolina). Comments by Ronald Zalesny (USDA Forest Service, North Central Research Station, Rhinelander, WI), Aurelien Sallé (University of Wisconsin, Madison, WI), and Randy Rousseau (MeadWestvaco Corp., Wickliffe, KY) greatly improved this manuscript. We thank the various suppliers for providing plant material. Thanks to John Blake and the members of the USDA Forest Service-Savannah River Engineering 
Division for infrastructure work at D-Area. Stan Zarnoch (USDA Forest Service, Asheville, NC) provided valuable statistical discussions and assistance. Funding was provided by the Department of Energy-Savannah River Operations Office through the US Forest Service-Savannah River under Interagency Agreement DE-AI09-00SR22188 and the US National Institute for Environmental Health Science (Grant No. P42 ES04696).

\section{References}

[1] Fenning TM, Gershenzon J. Where will the wood come from? Plantation forests and the role of biotechnology. Trends in Biotechnology 2002;20:291-6.

[2] Gladstone WT, Ledig FT. Reducing pressure on natural forests through high-yield forestry. Forest Ecology and Management 1990; 35:69-78.

[3] Isebrands JG, Karnosky DF. Environmental benefits of poplar culture. In: Dickmann DI, Isebrands JG, Eckenwalder JE, Richardson J, editors. Poplar Culture in North America. Ottawa, Canada: National Research Council; 2001. p. 207-18.

[4] Volk TA, Verwijst T, Tharakan PJ, Abrahamson LP, White EH. Growing fuel: a sustainability assessment of willow biomass crops. Frontiers in Ecology 2004;2:411-8.

[5] Dickmann DI, Stuart KW. The Culture of Poplars in Eastern North America. East Lansing, Michigan: Michigan State University; 1983 $168 \mathrm{p}$.

[6] Riemenschneider DE, Stanton BJ, Vallée G, Périnet P. Poplar breeding strategies. In: Dickmann DI, Isebrands JG, Eckenwalder JE, Richardson J, editors. Poplar Culture in North America. Ottawa, Canada: National Research Council; 2001. p. 43-76.

[7] Loehle C, Namkoong G. Constraints on tree breeding: growth tradeoffs, growth strategies, and defensive investments. Forest Science 1987;33:1089-97.

[8] Ostry ME, Berguson WE. Selecting hybrid poplars to reduce disease risk may also reduce biomass yield. Tree Planters' Notes 1993;44: 128-31.

[9] Chambers PGS, Borralho NMG. Importance of survival in shortrotation tree breeding programs. Canadian Journal of Forest Research 1997;27:911-7.

[10] Zalesny RS, Hall RB, Bauer EO, Riemenschneider DE. Shoot position affects root initiation and growth of dormant unrooted cuttings of Populus. Silvae Genetica 2003;52:273-9.

[11] Robison DJ, Raffa KF. Productivity, drought tolerance and pest status of hybrid Populus: tree improvement and silvicultural implications. Biomass and Bioenergy 1998;14:1-20.

[12] Tharakan PJ, Robison DJ, Abrahamson LP, Nowak CA. Multivariate approach for integrated evaluation of clonal biomass production potential. Biomass and Bioenergy 2001;21:237-47.

[13] Netzer DA, Tolsted DN, Ostry ME, Isebrands JG, Riemenschneider DE, Ward KT. Growth, yield, and disease resistance of 7- to 12-yearold poplar clones in the north central United States. St. Paul, MN U.S. Department of Agriculture, Forest Service, North Central Research Station; 2002. Gen. Tech. Rep. NC-229, p. 1-31.

[14] Nowak JT, Berisford CW. Effects of intensive forest management practices on insect infestation levels and loblolly pine growth. Environmental Entomology 2000;93:336-41.

[15] Coyle DR, McMillin JD, Hall RB, Hart ER. Cottonwood leaf beetle (Coleoptera: Chrysomelidae) defoliation impact on Populus growth and above-ground volume in a short-rotation woody crop plantation. Agricultural and Forest Entomology 2002;4:293-300.

[16] Newcombe G. The specificity of fungal pathogens of Populus. In Dickmann DI, Isebrands JG, Eckenwalder JE, Richardson J, editors. Poplar Culture in North America. Ottawa, Canada: National Research Council; 2001. p. 223-46.
[17] Mattson WJ, Hart ER, Volney WJA. Insect pests of Populus: coping with the inevitable. In: Dickmann DI, Isebrands JG, Eckenwalder JE, Richardson J, editors. Poplar Culture in North America. Ottawa, Canada: National Research Council; 2001. p. 219-48.

[18] Weiland JE, Stanosz JC, Stanosz GR. Prediction of long-term canker disease damage from the responses of juvenile poplar clones to inoculation with Septoria musiva. Plant Disease 2003;87:1507-14.

[19] Coyle DR, Nebeker TE, Hart ER, Mattson Jr WJ. Biology and management of insect pests in North American intensively managed hardwood forest systems. Annual Review of Entomology 2005;50: $1-29$.

[20] Coyle DR, McMillin JD, Kruse SC, Hart ER. Laboratory and field evaluations of two Bacillus thuringiensis formulations, Novodor and Raven, for control of cottonwood leaf beetle (Coleoptera: Chrysomelidae). Journal of Economic Entomology 2000;93:713-20.

[21] Coyle DR, McMillin JD, Hall RB, Hart ER. Deployment of tree resistance to insects in short-rotation Populus plantations. In: Wagner MR, Clancy KM, Lieutier F, Paine TD, editors. Mechanisms and Deployment of Resistance in Trees to Insects. New York: Kluwer Academic Publishers; 2002 [332pp]

[22] Coyle DR, Amrine Jr JW. New collection records and host range of the cottonwood leafcurl mite, Tetra lobulifera (Keifer) (Acari: Eriophyidae), in the USA. International Journal of Acarology 2004; 30:3-8.

[23] Coyle DR. Effects of clone, silvicultural, and miticide treatments on cottonwood leafcurl mite (Acari: Eriophyidae) damage in plantation Populus. Environmental Entomology 2002;31:1000-8.

[24] Newcombe G, Chastagner GA, Schutte W, Stanton BJ. Mortality among hybrid poplar clones in a stool bed following leaf rust caused by Melampsora medusae f.sp. deltoidae. Canadian Journal of Forest Research 1994;24:1984-7.

[25] Royle DJ, Ostry ME. Disease and pest control in the bioenergy crops poplar and willow. Biomass and Bioenergy 1995;9:69-79.

[26] Steenackers J, Steenackers M, Steenackers V, Stevens M. Poplar diseases, consequences on growth and wood quality. Biomass and Bioenergy 1996;10:267-74.

[27] Farmer RE, Wilcox JR. Preliminary testing of eastern cottonwood clones. Theoretical and Applied Genetics 1968;38:197-201.

[28] Olson JR, Jourdain CJ, Rousseau RJ. Selection for cellulose content, specific gravity, and volume in young Populus deltoides clones. Canadian Journal of Forest Research 1985;15:393-6.

[29] WSRC. Treatability study work plan for phytoremediation of the DArea TCE-contaminated groundwater plume (U). WSRC-RP-20004015, Revision 1 Redline. Savannah River Site, Aiken, South Carolina: Westinghouse Savannah River Company; 2000 [37pp].

[30] Coleman MD, Coyle DR, Blake J, Britton K, Buford M, Campbell B, et al. Production of short rotation woody crops grown with a range of nutrient and water availability: establishment report and first-year responses. Asheville, NC: USDA-Forest Service, Southern Research Station; 2004. SRS-GTR-072, p. 1-21.

[31] Coyle DR, Coleman MD, Durant JA, Newman LA. Survival and growth of 31 Populus clones in South Carolina. Biomass and Bioenergy, this issue, doi:10.1016/j.biombioe.2005.08.005.

[32] DesRochers A, Thomas BR. A comparison of pre-planting treatments on hardwood cuttings of four hybrid poplar clones. New Forests 2003;26:17-32

[33] Fang Y, Hart ER. Effect of cottonwood leaf beetle (Coleoptera: Chrysomelidae) larval population levels on Populus terminal damage. Environmental Entomology 2000;29:43-8.

[34] Larson PR, Isebrands JG. The plastochron index as applied to developmental studies of cottonwood. Canadian Journal of Forest Research 1971:1:1-11.

[35] Schreiner EJ. Rating poplars for Melampsora leaf rust infection. Minneapolis, MN: USDA-Forest Service, North Central Research Station; 1959. Res. Note NE-90, p. 1-3.

[36] Ostry ME, McNabb HS. Susceptibility of Populus species and hybrids to disease in the north central United States. Plant Disease 1985; 69:755-7. 
[37] Abrahamson LP, White EH, Nowak CA, Biggs RD, Robison DJ. Evaluating hybrid poplar clonal growth potential in a three-year-old genetic selection field trial. Biomass 1990;21:101-14.

[38] Robison DJ, Raffa KF. Characterization of hybrid poplar clones for resistance to the forest tent caterpillar. Forest Science 1994;40:686-714.

[39] Caldbeck ES, McNabb HS, Hart ER. Poplar clonal preferences of the cottonwood leaf beetle. Journal of Economic Entomology 1978;71: 518-20.

[40] Newcombe G, Chastagner GA. A leaf rust epidemic of hybrid poplar along the Lower Columbia River caused by Melampsora medusae. Plant Disease 1993;77:528-31.

[41] Larsson S. Stressful times for the plant stress-insect performance hypothesis. Oikos 1989;56:277-83.

[42] Koricheva J, Larsson S, Haukioja E. Insect performance on experimentally stressed woody plants: a meta-analysis. Annual Review of Entomology 1998;43:195-216.
[43] Huberty AF, Denno RF. Plant water stress and its consequences for herbivorous insects: a new synthesis. Ecology 2004;85:1383-98.

[44] Daane KM, Williams LE. Manipulating vineyard irrigation amounts to reduce insect pest damage. Ecological Applications 2003;13: 1650-66.

[45] Ares A. Changes through time in traits of poplar clones in selection trials. New Forests 2002;23:105-19.

[46] Lo MH, Abrahamson LP. Principal component analysis to evaluate the relative performance of nine year old hybrid poplar clones. Biomass and Bioenergy 1996;10:1-6.

[47] Yamasaki M, Kikuzawa K. Temporal and spatial variations in leaf herbivory within a canopy of Fagus crenata. Oecologia 2003;137: 226-32.

[48] Coyle DR, McMillin JD, Hall RB, Hart ER. Cottonwood leaf beetle (Coleoptera: Chrysomelidae) larval performance on eight Populus clones. Environmental Entomology 2001;30:748-56. 